hard Serexhe und Peter Weibel (2007). Der Aufsatz „,Du lebloses, verdammtes Automat!'. Unser Schicksal vis-à-vis Maschinen und Automaten ..." des ausgewiesenen Descartes-Experten Hans-Peter Schütt zur ideengeschichtlichen Vertiefung des Automaten-Motivs kam sicherlich zu spät, um Berücksichtigung zu finden. Was man sich abschließend für den gehobenen Preis hätte wünschen können, wäre ein besserer Bilddruck gewesen - wenn nicht auch ein gut gebundenes Hardcover für dieses wunderbare Buch eine bessere Form geboten hätte. Der Band gewann den Förderpreis der (ehemaligen) Deutschen Gesellschaft für Geschichte der Medizin, Naturwissenschaft und Technik (DGGMNT) und nach der anregenden Lektüre bleibt festzuhalten: zu Recht.

Funding Open Access funding enabled and organized by Projekt DEAL.

Open Access Dieser Artikel wird unter der Creative Commons Namensnennung 4.0 International Lizenz veröffentlicht, welche die Nutzung, Vervielfältigung, Bearbeitung, Verbreitung und Wiedergabe in jeglichem Medium und Format erlaubt, sofern Sie den/die ursprünglichen Autor(en) und die Quelle ordnungsgemäß nennen, einen Link zur Creative Commons Lizenz beifügen und angeben, ob Änderungen vorgenommen wurden.

Die in diesem Artikel enthaltenen Bilder und sonstiges Drittmaterial unterliegen ebenfalls der genannten Creative Commons Lizenz, sofern sich aus der Abbildungslegende nichts anderes ergibt. Sofern das betreffende Material nicht unter der genannten Creative Commons Lizenz steht und die betreffende Handlung nicht nach gesetzlichen Vorschriften erlaubt ist, ist für die oben aufgeführten Weiterverwendungen des Materials die Einwilligung des jeweiligen Rechteinhabers einzuholen.

Weitere Details zur Lizenz entnehmen Sie bitte der Lizenzinformation auf http://creativecommons.org/ licenses/by/4.0/deed.de.

\title{
Todd, David: A Velvet Empire. French Informal Imperialism in the Nineteenth Century, 368 S., Princeton UP, Princeton, NJ u.a. 2021.
}

\section{Jürgen Osterhammel}

Angenommen: 30. Juni 2021 / Online publiziert: 4. August 2021

(C) Der/die Autor(en) 2021

Auch in der buchbetonten Geschichtswissenschaft können gelegentlich Aufsätze klassischen Rang erreichen. 1953 veröffentlichten zwei junge Dozenten, John Gallagher und Ronald Robinson, später einflussreiche Professoren in Cambridge und

\footnotetext{
Jürgen Osterhammel $(\varangle)$

Albert-Ludwigs-Universität Freiburg im Breisgau, Freiburg i. Br., Deutschland

E-Mail: juergen.osterhammel@ frias.uni-freiburg.de
} 
Oxford, den gerade einmal fünfzehn Seiten langen Artikel „The Imperialism of Free Trade“. Unter anderem wurde darin der Unterschied zwischen formal empire und informal empire entwickelt. Als informell werden politische Strategien und daraus resultierende Strukturen bezeichnet, bei denen imperiale Akteure unter der Voraussetzung asymmetrischer Machtverhältnisse ihre Interessen durch juristische Privilegiensicherung und Einbeziehung örtlicher Kollaborateure zu wahren verstehen, ohne den kostspieligen Aufwand offener Kolonialherrschaft treiben zu müssen. Gallagher und Robinson sprachen von ,empire on the cheap“, sahen den britischen ,imperialism of free trade“ zwischen etwa 1830 und 1880 als die deutlichste Ausprägung solch aggressiver Einflusspolitik und formulierten die Hypothese, Imperialmächte zögen generell informelle Arrangements vor und griffen erst in Krisensituationen zum radikaleren Mittel des ,imperial takeover“.

David Todd wendet die bewährte, doch heute manchmal zu sehr auf soft power eingeschränkte Kategorie des informal empire nicht einfach auf sein Thema an. Er denkt sie weiter, schneidert sie auf seinen Gegenstand zu, sucht sie sinngemäß in den Quellen und garniert sie mit einprägsamen Umschreibungen. „A Velvet Empire“" wird man als Buchtitel nicht so schnell vergessen, ist aber nur bedingt eine glückliche Wahl. Der Titel spielt auf eines der fünf Kapitel an, in dem gezeigt wird, wie sich die französische Wirtschaft in den mittleren Jahrzehnten des 19. Jahrhunderts als Exporteurin gehobener Bürgerlichkeit und der damit verbundenen Produkte - auch Samt und Lyoner Seide - auf den Weltmärkten präsentierte, während man buchstäblich glanzlosere Warengattungen wie Baumwolltextilien oder Maschinen der britischen Konkurrenz überließ.

Gemeint ist mit „Samtimperium“ hingegen keineswegs, dass der französische Imperialismus mit Samthandschuhen vorgegangen wäre. Ganz im Gegenteil. Als nach einem Jahrzehnt des Experimentierens mit dünner Besatzung (occupation restreinte), also einer militärischen Form von Informalität, die labilen Kompromisse mit einheimischen Eliten zusammenbrachen, wurde Algerien zwischen 1840 und 1847 zum Schauplatz eines Eroberungskriegs der schlimmsten Sorte. Ein Vierteljahrhundert später, als das Osmanische Reich und Ägypten durch schleichende Finanzkontrolle gefügig und profitabel gemacht werden sollten, übertrafen französische Diplomaten, Bankiers und Geschäftsleute ihre britischen Kollegen an unerbittlichem Durchsetzungswillen. Von „,imperialism light“ kann also keine Rede sein. Und auch das charmante Konstrukt eines „champagne capitalism“ (S. 123) weist in eine falsche Richtung. Solche wortschöpferische Pfiffigkeit beschert uns nur einen weiteren der heute beliebten Bindestrich-Kapitalismen. Sie alle suchen hinter den meist nur zaghaft theoretisierten Zusammenhängen der eigentlichen Kapitallogik ein selbst nicht erklärungsbedürftiges primum movens. Hier also der Durst der Betuchten aller Länder nach dem Schaumwein der Witwe Clicquot?

So einfach ist es bei David Todd zum Glück nun doch nicht, und es wird höchste Zeit zu sagen, dass dies ein klug konzipiertes, einfallsreich und sorgfältig recherchiertes und in flottem Argumentationstempo geschriebenes Buch ist. $\mathrm{Zu}$ seinen Stärken gehört die Einbindung der Wirtschaft- und Finanzgeschichte, auch moderat quantitativer Darstellungsweisen, in verschiedenste Nachbarbereiche von der Geschichte des ökonomischen Denkens (das gerade in Frankreich vielfach eine Sache von Politikern und Geschäftsleuten war) bis zur keineswegs marginalen Frage der 
exterritorialen Rechtsstellung von Ausländern im - vor der britischen Okkupation von 1882 - informell durchdrungenen Ägypten.

Bei allem Detailreichtum entwirft David Todd ein kohärentes Bild: Nach dem Kollaps von Napoleons Reich brach keineswegs eine Periode konservativer Imperialabstinenz und liberaler Imperialfeindschaft aus. Vielmehr traten ältere Vorstellungen, vor allem verkörpert von dem langlebigen Strippenzieher Charles-Maurice de Talleyrand, hervor, die Frankreichs Einfluss in der Welt stärken und erweitern wollten. Diese Bestrebungen einten alle maßgebenden politischen Kräfte und zogen sich im Grunde über die verschiedenen Systemwechsel bis in die späten 1870er Jahre hin. Danach konsolidierte sich endgültig die Dritte Republik, eine relativ stabile franko-britische Beziehung zerbrach 1882 über Ägypten, und Frankreichs charakteristisches imperiales Geschäftsmodell ging in einem weltweiten Trend auf, den Todd mit Charles Maier als „Territorialisierung“ versteht. Frankreich wurde zu einer Standardkolonialmacht unter vielen anderen.

Das Geschäftsmodell beruhte auf der produktiven Kraft der französischen Wirtschaft, die hinter der britischen keineswegs zweitklassig war, auf der erfolgreichen Kultivierung des globalen Luxusmarkts sowie auf einer hohen Sparquote, wie sie überhaupt erst den Einsatz von Kapitalexport als politischem Instrument ermöglichte. Der französische Weg war deutlich informeller als der einzige andere, der als Maßstab zählte: der britische. Formeller Kolonialbesitz war unbedingt zu vermeiden, ebenso jegliche Kolonisation durch unkontrollierbare Privatpioniere; erst 1873 erhielten die Siedler in Algerien freie Hand. Todd erklärt „France's debacle“ (S. 279) eine Spur weniger elegant als den Erfolg des Modells nach 1815. Warum ließ sich das Momentum unter republikanischen Vorzeichen nicht aufrechterhalten? Hybris (,overreach“, S. 218), politische Handwerksfehler und schlichtweg Pech scheinen ausschlaggebend gewesen zu sein. All dies kam beim Scheitern der Intervention in Mexiko 1867 zusammen. Die Marionette Napoleons III., der habsburgische Kaiserbruder Maximilian, endete vor einem mexikanischen Hinrichtungspeloton.

Funding Open Access funding enabled and organized by Projekt DEAL.

Open Access Dieser Artikel wird unter der Creative Commons Namensnennung 4.0 International Lizenz veröffentlicht, welche die Nutzung, Vervielfältigung, Bearbeitung, Verbreitung und Wiedergabe in jeglichem Medium und Format erlaubt, sofern Sie den/die ursprünglichen Autor(en) und die Quelle ordnungsgemäß nennen, einen Link zur Creative Commons Lizenz beifügen und angeben, ob Änderungen vorgenommen wurden.

Die in diesem Artikel enthaltenen Bilder und sonstiges Drittmaterial unterliegen ebenfalls der genannten Creative Commons Lizenz, sofern sich aus der Abbildungslegende nichts anderes ergibt. Sofern das betreffende Material nicht unter der genannten Creative Commons Lizenz steht und die betreffende Handlung nicht nach gesetzlichen Vorschriften erlaubt ist, ist für die oben aufgeführten Weiterverwendungen des Materials die Einwilligung des jeweiligen Rechteinhabers einzuholen.

Weitere Details zur Lizenz entnehmen Sie bitte der Lizenzinformation auf http://creativecommons.org/ licenses/by/4.0/deed.de. 